\section{PROPOSTA E VALIDAÇÃO DE UM PROTOCOLO DE TRIAGEM PARA IDENTIFICAR AS MANIFESTAÇÕES FONOAUDIOLÓGICAS NA HANSENÍASE}

\author{
Proposal and validation of a screening Protocol for Identifying \\ Speech, Language and Hearing Manifestations in Leprosy
}

\begin{abstract}
Propuesta y validación de un Protocolo de Selección para la Identificación de Manifestaciones Fonoaudiológicas de la Lepra
\end{abstract}

\section{RESUMO}

Objetivo: Propor e validar o conteúdo do protocolo de triagem para identificação das manifestações fonoaudiológicas na hanseníase. Métodos: Este estudo foi organizado em duas fases: pesquisa bibliográfica para subsidiar a construção do instrumento de triagem e posterior avaliação da adequação do conteúdo por um painel de especialistas. Para avaliar a relevância/ representatividade das respostas, utilizou-se o Índice de Validade de Conteúdo (IVC) com concordância mínima de $90 \%$. Resultados: A leitura do material resultou na construção de um instrumento de 18 itens, abrangendo quatro seções: audiologia, voz, deglutição e fala/motricidade orofacial. A etapa seguinte constou da análise da representatividade dos itens do protocolo pelos juízes. Após a primeira análise, a validação do conteúdo resultou na permanência de dez com IVC total de $100 \%$. Conclusão: O protocolo de triagem foi considerado um instrumento válido para identificar as manifestações fonoaudiológicas na hanseníase.

Descritores: Hanseníase; Fonoaudiologia; Estudos de Validação; Atenção Primária à Saúde.

\section{ABSTRACT}

Objective: To propose and validate the content of a screening protocol for identifying speech, language and hearing manifestations in leprosy. Methods: This study was organized into two phases: bibliographic research to support the development of the screening instrument and subsequent evaluation of the adequacy of the content by a panel of experts. The relevance/representativeness of the responses was evaluated using the Content Validity Index (CVI) with a minimum agreement of $90 \%$. Results: The reading of the material resulted in the development of an instrument with 18 items covering four sections: audiology, voice, swallowing and speech/orofacial motricity. The next phase consisted of the analysis of the representativeness of the protocol items by the judges. After the first analysis, the content validation resulted in ten items with CVI of 100\%. Conclusion: The screening protocol was considered a valid instrument for the identification of speech, language and hearing manifestations in leprosy.

Descriptors: Leprosy; Speech, Language and Hearing Sciences; Validation Studies; Primary Health Care.
Artigo Original

Loise Elena Zanin ${ }^{(1)}$

Daniel Hardy Melo ${ }^{(2)}$

Maria do Socorro Melo

Carneiro $^{(3)}$

Juliana Mendes Gomes ${ }^{(1)}$

Vicente de Paulo Teixeira Pinto ${ }^{(2)}$

Leonardo Wilner Barros Silva ${ }^{(2)}$

Ítalo Rosse Santos ${ }^{(2)}$

Amanda Kathleen Mendonça

Rodrigues $^{(2)}$

1) Hospital Regional Norte - HRN - Sobral

- CE - Brasil

2) Universidade Federal do Ceará - UFC -

Sobral - CE - Brasil

3) Universidade Estadual Vale do Acaraú UVA - Sobral - CE - Brasil

Recebido em: 03/05/2016 Revisado em: 13/07/2016 Aceito em: 29/10/2016 


\section{RESUMEN}

Objetivo: Proponer y validar el contenido del protocolo de selección para la identificación de las manifestaciones fonoandiológicas de la lepra. Métodos: Este estudio fue organizado en dos fases: la investigación bibliográfica para subsidiar la construcción del instrumento de selección seguida de la evaluación de la adecuación del contenido a través de un panel de especialistas. Para valorar la relevancia/representatividad de las respuestas se utilizó el Índice de Validad de Contenido (IVC) con concordancia mínima del 90\%. Resultados: La lectura del material ha resultado en la construcción de un instrumento de 18 items involucrando cuatro secciones: audiología, voz, deglución y habla/motricidad orofacial. La etapa siguiente fue el análisis de la representatividad de los items del protocolo de parte de los jueces. Tras el primer análisis la validación del contenido ha resultado en la permanencia de diez con IVC totalizando el 100\%. Conclusión. El protocolo de selección fue considerado un instrumento válido para la identificación de las manifestaciones fonoaudiológicas de la lepra.

Descriptores: Lepra; Fonoaudiología; Estudios de Validación; Atención Primaria de Salud.

\section{INTRODUÇÃO}

A atuação da fonoaudiologia na Atenção Primária à Saúde (APS) é recente. Com trajetória ainda em construção, faz-se necessário o investimento em pesquisas de forma a nortear a prática e consolidar sua atuação na Estratégia Saúde da Família (ESF) baseada em evidências, contribuindo com a qualidade de vida da população ${ }^{(1)}$.

É importante o envolvimento do fonoaudiólogo nos programas estabelecidos pelo Ministério da Saúde visando fortalecer a capacidade de resposta do sistema de saúde às doenças emergentes e endemias, por exemplo, o controle da hanseníase ${ }^{(2)}$.

A hanseníase é uma moléstia de evolução prolongada com grande potencial incapacitante e é considerada um importante problema de saúde pública. Suas alterações podem comprometer significativamente a voz, a audição e as funções estomatognáticas, sendo necessária a intervenção fonoaudiológica ${ }^{(3)}$.

O diagnóstico da doença na APS é, essencialmente, clínico. $\mathrm{Na}$ anamnese, deve ser abordada a presença dos sinais e sintomas, bem como acolher as dúvidas, impressões e opiniões do usuário. $\mathrm{O}$ acolhimento deve ser pautado no diálogo e respeito, e os profissionais precisam ser capacitados para isso. O primeiro atendimento é fundamental para estabelecer essa relação entre usuário e profissional, para isso, a abordagem deve estimular a troca de ideias acerca da doença, crenças e preconceitos, forma de contágio, cura e importância do envolvimento da família em um possível tratamento, incluindo o exame dos contatos ${ }^{(4)}$.

Neste contexto, considerando-se que a hanseníase ainda é um desafio em saúde pública no território brasileiro devido às altas taxas de detecção em que APS possui um papel de extrema importância para o controle da doença, torna-se necessária a utilização de instrumentos que permitam avaliar o alcance dessa estratégia ${ }^{(5)}$.

Sendo assim, a participação efetiva do fonoaudiólogo nas equipes multidisciplinares da APS e, consequentemente, no acompanhamento dos pacientes com hanseníase, possibilitaria aos usuários uma melhor qualidade de vida por meio da identificação e intervenção em aspectos comumente ignorados. Dessa forma, existe a necessidade da inclusão do fonoaudiólogo no atendimento desses indivíduos, bem como a construção de instrumentos que o auxiliem em sua atuação nesse cenário e no fortalecimento dos programas desenvolvidos nos centros de saúde da família (CSF).

Diante disso, este estudo teve como objetivo propor e validar o conteúdo do protocolo de triagem para identificação das manifestações fonoaudiológicas na hanseníase.

\section{MÉTODOS}

O estudo foi do tipo observacional, descritivo e transversal, com abordagem quantitativa. A pesquisa ocorreu na cidade de Sobral-CE, sendo realizada a revisão da literatura e elaboração do protocolo no período de janeiro a março de 2016, e a validação do protocolo pelos juízes ocorreu no período de abril a maio de 2016.

O protocolo foi elaborado com base na literatura nacional e internacional, pautado em estudos que abordavam as manifestações fonoaudiológicas na hanseníase. Assim, no primeiro momento, realizou-se uma busca na Biblioteca Virtual de Saúde (BVS), incluindo resumos/abstracts de artigos publicados nos últimos 20 anos (1995 a 2015) de periódicos de acesso livre ou não. Foram utilizadas as seguintes combinações de palavras-chave para a busca na base de dados: "hanseníase e voz", "hanseníase e audição", "hanseníase e cavidade oral", "hanseníase e paralisia facial".

Os critérios de inclusão adotados para a seleção das referências foram: artigos em inglês, português e espanhol sobre manifestações fonoaudiológicas na hanseníase. Como critérios de exclusão, adotou-se: publicações duplicadas, editoriais, boletins epidemiológicos e estudos que tangenciaram a temática. A busca inicial resultou em 66 pesquisas, porém, após a aplicação dos critérios de elegibilidade do estudo, apenas 18 publicações contribuíram de forma efetiva na elaboração da primeira versão do protocolo. 
No segundo momento, foi utilizado o modelo de validação dos testes psicométricos, o qual prevê a integração de várias evidências de validade que, juntamente com a teoria, possam sustentar a interpretação pretendida aos escores de um teste para uso específico ${ }^{(5,6)}$.

Para quantificar o grau de concordância acerca da relevância do conteúdo do instrumento de triagem, utilizouse a abordagem que envolve a seleção de um painel de especialistas. Os critérios de inclusão para compor o painel foram: profissionais da categoria de fonoaudiologia com experiência acima de três anos na área da ESF e/ou fonoaudiólogos com publicação de trabalhos científicos na área de ESF. Os critérios de exclusão adotados foram: fonoaudiólogos que não participaram da segunda etapa de revalidação do protocolo, não entrega dos questionários dentro do prazo preestabelecido ou realização de preenchimento inadequado dos questionários.

Para validação de conteúdo são necessários, no mínimo, três juízes especialistas e um painel com mais de dez provavelmente será desnecessário ${ }^{(5)}$. Assim, buscou-se selecionar 10 possíveis juízes de várias regiões do Brasil. Na pesquisa por trabalhos que abordavam a atuação da fonoaudiologia na ESF, foram encontrados 10 artigos de diferentes regiões do país. O primeiro autor de cada artigo recebeu um e-mail convidando-o a participar do painel de juízes. Contudo, apenas 2 pesquisadores da região nordeste responderam ao convite.

Em virtude do pequeno quantitativo de pesquisadores com publicação na área de ESF que responderam ao e-mail, o convite se estendeu a mais dois profissionais da região Nordeste, especificamente do Ceará, que atuavam há mais de três anos neste cenário. Assim, iniciou-se a etapa I de validação do conteúdo, com o envio para os juízes do Termo de Consentimento Livre e Esclarecido, de uma carta contendo os objetivos do trabalho, do instrumento de avaliação e do guia instrucional, com formulário para validação do conteúdo.

Para avaliar a relevância/representatividade das respostas por meio do Índice de Validade de Conteúdo (IVC), utilizou-se uma escala dicotômica de dois pontos visando a objetividade na coleta das respostas dos juízes, sendo $1=$ relevante ou representativo e $2=$ item não relevante ou não representativo. Os itens que receberem pontuação " 2 ” foram retirados. Como o painel de especialistas era composto por apenas quatro juízes, todos precisavam concordar com o item analisado para este ser considerado representativo ${ }^{(5)}$.

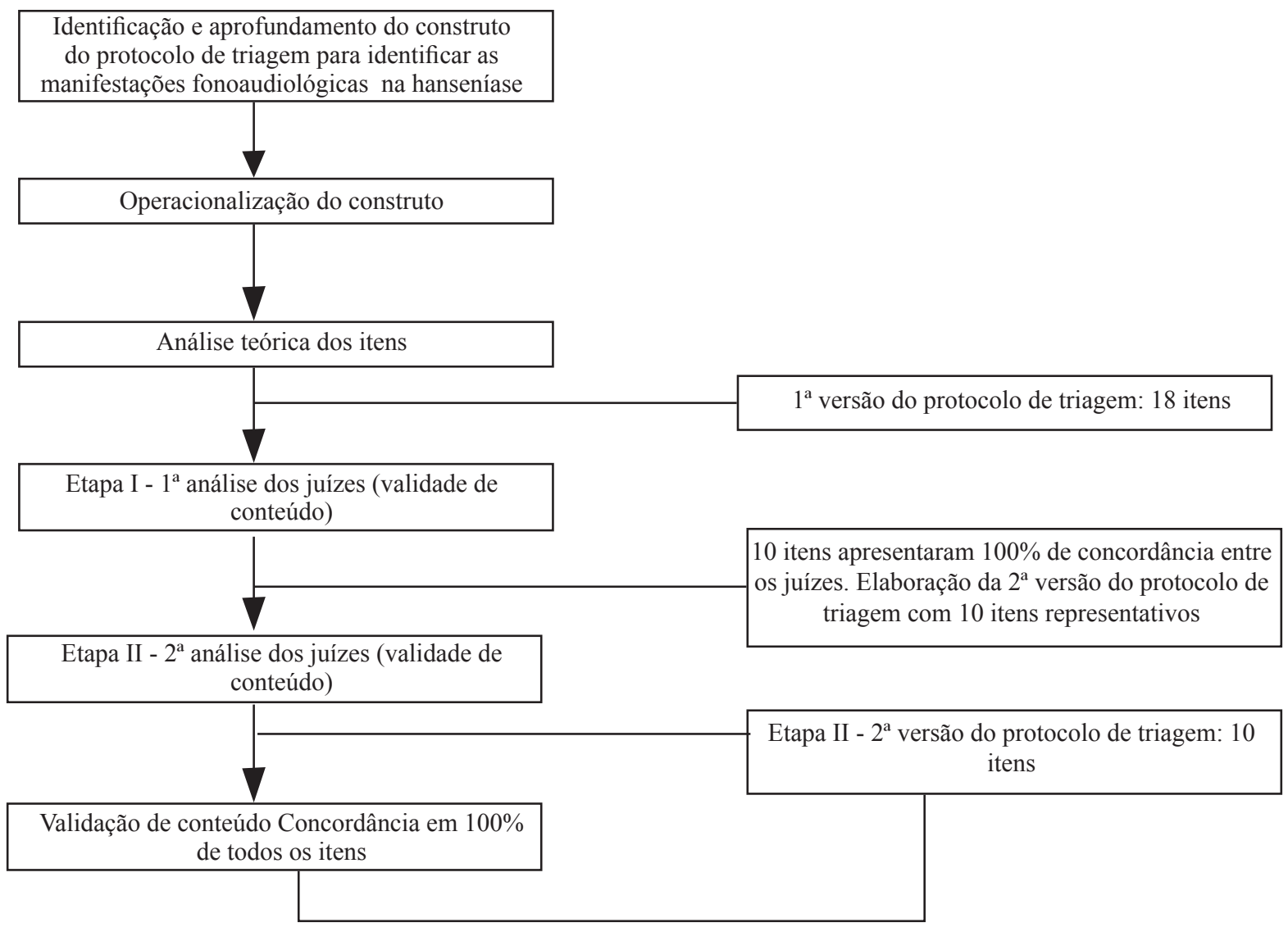

Figura 1 - Representação gráfica das etapas de validação do protocolo de triagem. Sobral, Ceará, 2016. 


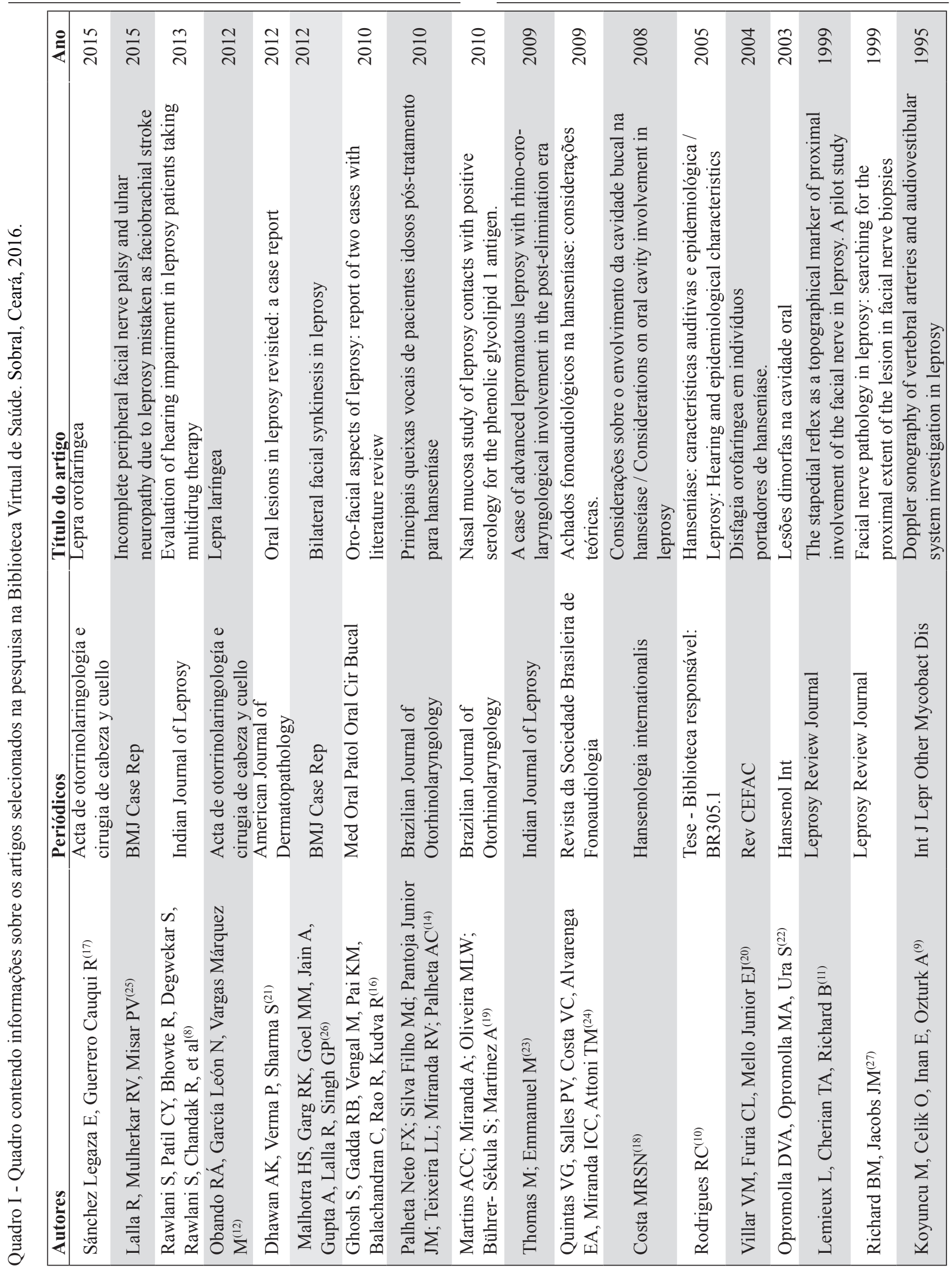


Figura 2 - Primeira versão do protocolo de triagem para identificar as manifestações fonoaudiológicas na hanseníase. Sobral, Ceará, 2016.

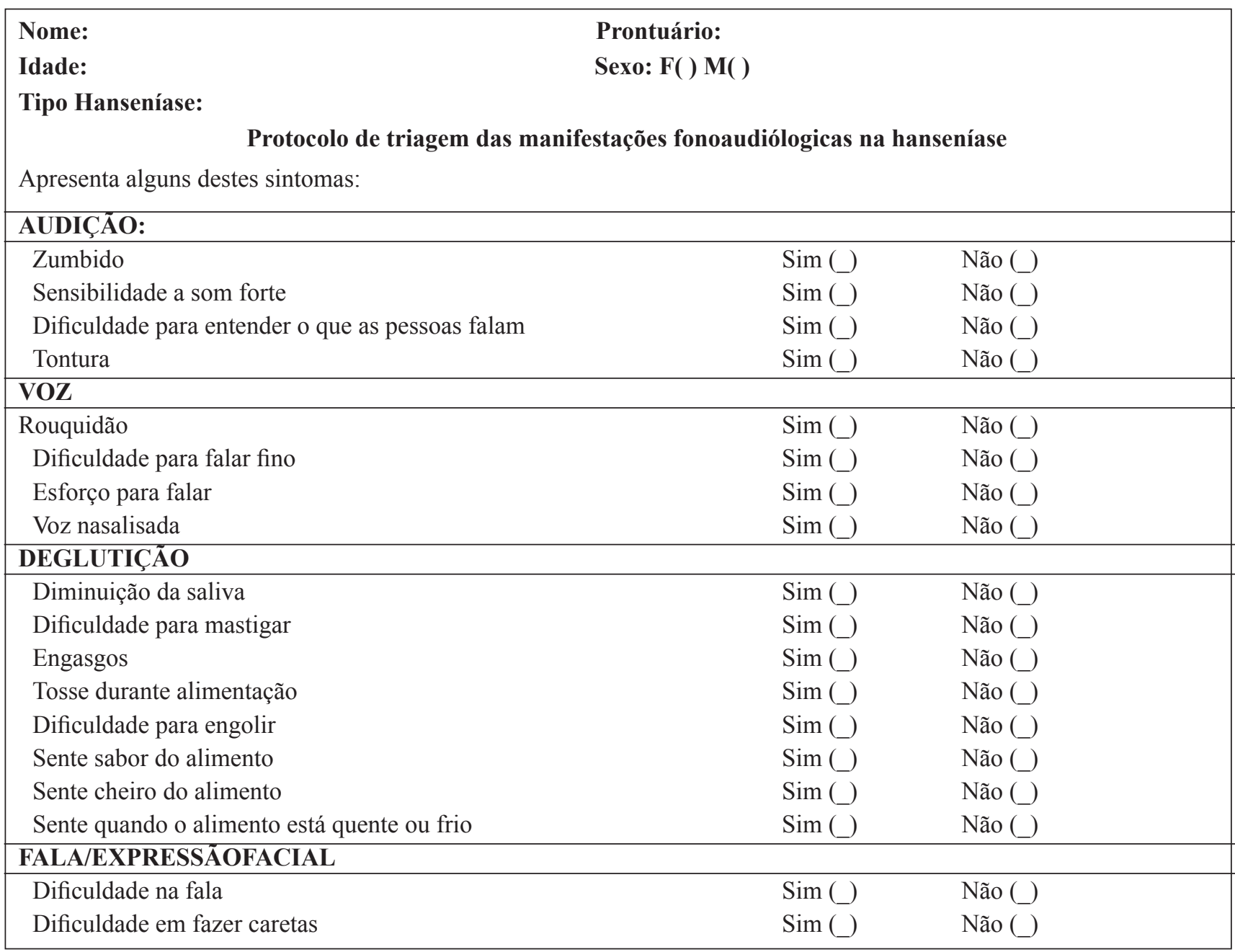

Tabela I - Caracterização do painel de juízes. Sobral, Ceará, 2016.

\begin{tabular}{lcccc}
\hline Variáveis & Juiz 1 & Juiz 2 & Juiz 3 & Juiz 4 \\
\hline Idade & 31 anos & 37 anos & 38 anos & Não informado \\
Sexo & Masculino & Feminino & Feminino & Feminino \\
Formação & Especialista & Especialista & Especialista & Especialista \\
Tempo de formação & 8 anos & 13 anos & 12 anos & 7 anos \\
& NASF*/ & NASF*/ & & \\
Atuação profissional & Assistência & Assistência & NASF* / Clínica & Clínica \\
& Hospitalar e & & \\
Experiência em ESF & 8 anos & Domiciliar & 10 anos & Sem atuação \\
Publicação da área & Não & 13 anos & Não & Sim \\
\hline *NASF: Nú́ceo de A & Sim
\end{tabular}

*NASF: Núcleo de Apoio à Saúde da Família; ESF: Estratégia Saúde da Família. 
Tabela II - Índice de Velocidade de Conteúdo do protocolo de triagem para identificar as manifestações fonoaudiológicas na hanseníase - Etapa I. Sobral, Ceará, 2016.

\begin{tabular}{lcc}
\hline Itens do instrumento de validação e guia instrucional & $\begin{array}{c}\text { Número de juízes em } \\
\text { concordância (n=04) }\end{array}$ & $\begin{array}{c}\text { Representativo/Não } \\
\text { representativo }\end{array}$ \\
\hline AUDIÇÃO & 4 & Representativo \\
Zumbido & 3 & Não representativo \\
Sensibilidade a som forte & 3 & Não representativo \\
Dificuldade para entender o que as pessoas falam & 3 & Não representativo \\
Tontura & 4 & Representativo \\
VOZ & 3 & Não representativo \\
Rouquidão & 4 & Representativo \\
Dificuldade para falar fino & 3 & Não representativo \\
Esforço para falar & & \\
Voz nasalisada & 3 & Não representativo \\
DEGLUTIÇÃO & 4 & Representativo \\
Diminuição da saliva & 4 & Representativo \\
Dificuldade para mastigar & 4 & Representativo \\
Engasgos & 4 & Representativo \\
Tosse durante alimentação & 3 & Não representativo \\
Dificuldade para engolir & 3 & Não representativo \\
Sente sabor do alimento & 4 & Representativo \\
Sente cheiro do alimento & & Representativo \\
Sente quando o alimento está quente ou frio & 4 & Representativo \\
FALA/EXPRESSÃO FACIAL & 4 & IVC total*=56\% \\
Dificuldade na fala & & Dificuldade em fazer caretas \\
Total: 10 questões representativas & &
\end{tabular}

*IVC total deve ser igual ou maior que $90 \%$

Tabela III - Índice de Validade de Conteúdo (IVC) do protocolo de triagem para identificar as manifestações fonoaudiológica na hanseníase. Sobral, Ceará, 2016.

\begin{tabular}{|c|c|c|}
\hline $\begin{array}{l}\text { Itens do instrumento de validação e guia } \\
\text { instrucional }\end{array}$ & $\begin{array}{l}\text { Número de juízes em } \\
\text { concordância }(n=04)\end{array}$ & $\begin{array}{c}\text { Representativo/ } \\
\text { Não representativo }\end{array}$ \\
\hline \multicolumn{3}{|l|}{ AUDIÇÃO } \\
\hline Zumbido & 4 & Representativo \\
\hline \multicolumn{3}{|l|}{ VOZ } \\
\hline Rouquidão & 4 & Representativo \\
\hline Esforço para falar & 4 & Representativo \\
\hline \multicolumn{3}{|l|}{ DEGLUTIÇÃO } \\
\hline Dificuldade para mastigar & 4 & Representativo \\
\hline Engasgos & 4 & Representativo \\
\hline Tosse durante alimentação & 4 & Representativo \\
\hline Dificuldade para engolir & 4 & Representativo \\
\hline Sente quando o alimento está quente ou frio & 4 & Representativo \\
\hline \multicolumn{3}{|l|}{ FALA/EXPRESSÃO FACIAL } \\
\hline Dificuldade na fala & 4 & Representativo \\
\hline \multirow[t]{2}{*}{ Dificuldade em fazer caretas } & 4 & Representativo \\
\hline & & IVC total $*=100 \%$ \\
\hline
\end{tabular}

* IVC total deve ser igual ou maior que $90 \%$ 
Figura 3 - Protocolo de triagem das manifestações fonoaudiológicas na hanseníase. Sobral, Ceará, 2016.

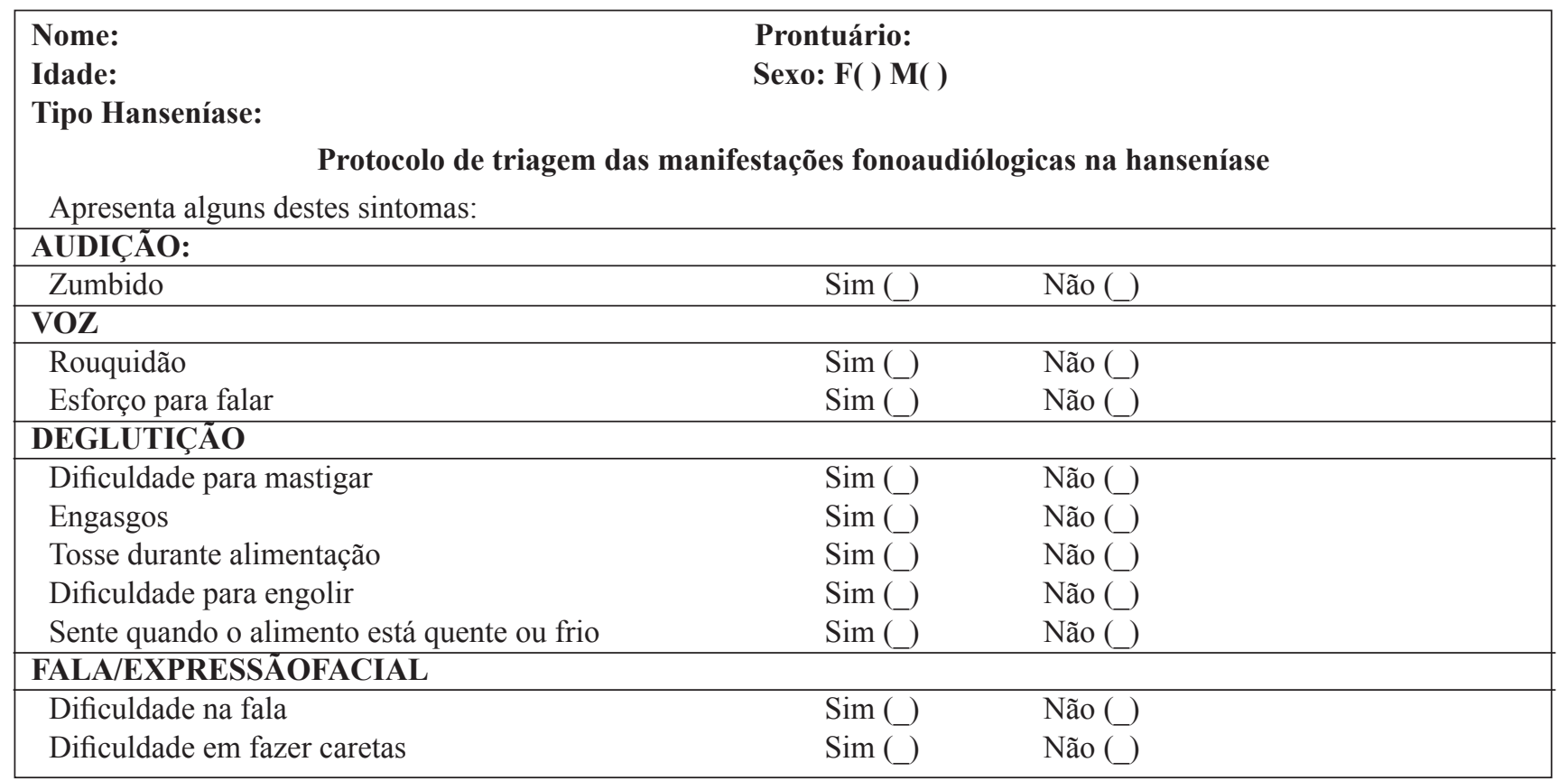

Após a devolução dos questionários preenchidos, as respostas dos juízes eram analisadas, obtendo-se os IVC. Assim, realizou-se uma revisão do instrumento de triagem, com consequente modificação do seu conteúdo, finalizando a etapa I de validação. Na etapa II, de revalidação do conteúdo, os questionários com aspectos revisados foram enviados aos mesmos juízes. A etapa II seguiu os mesmos critérios e análise da etapa I. A Figura 1 representa as etapas desta pesquisa.

Para verificar o IVC de uma forma geral, deverá haver uma concordância mínima de $90 \%$ ou mais ${ }^{(5,6)}$. Dessa forma, considerou-se esse valor para IVC entre os juízes para finalizar a etapa.

As etapas de elaboração e validação do protocolo estão descritas na Figura 1.

O presente estudo seguiu a Resolução CNS $n^{\circ}$. 466/2012, sendo submetido ao Comitê de Ética em Pesquisa da Universidade Federal do Ceará (CEP/UFC/PROPESQ), com aprovação dada pelo parecer $n^{\circ}$. 1.488.842.

\section{RESULTADOS}

A leitura e discussão do material descrito no Quadro I resultaram na construção de um instrumento de 18 itens, abrangendo quatro seções: audiologia (quatro itens); voz (quatro itens); deglutição (oito itens); e fala/motricidade orofacial (dois itens), que estão detalhados na Figura 2.
Após a definição do protocolo, iniciou-se a segunda etapa do estudo, ou seja, a análise dos juízes. A Tabela I apresenta a caracterização desses profissionais.

Os itens do instrumento de triagem foram avaliados quanto a sua representatividade. Posteriormente, as respostas dos especialistas foram analisadas quantitativamente, observando-se as proporções de concordância descritas na Tabela II.

Na validação do conteúdo, os examinadores analisaram os itens quanto à representatividade. A primeira avaliação resultou na retirada de oito itens julgados como não representativos. A análise não alterou as seções a serem avaliadas pelo protocolo.

A primeira etapa desse estudo resultou na modificação do instrumento de triagem, reduzindo os itens inicialmente propostos. Os juízes realizaram nova análise, revalidando o instrumento, que resultou na concordância e permanência dos dez itens considerados anteriormente como representativos. O IVC total de $100 \%$ pode ser identificado na Tabela III.

Assim, finalizou-se o protocolo de triagem e sua versão final está apresentada na Figura 3.

\section{DISCUSSÃO}

O perfil dos juízes que participaram desse estudo para validação do instrumento está de acordo com o que a literatura aconselha. Aapreciação do instrumento por parte de 
examinadores, experientes e competentes na área específica, é um recurso bastante utilizado, mas os especialistas devem ser escolhidos cuidadosamente e, portanto, representarem o que há de mais recente no conhecimento da área. Além disso, recomenda-se ter experiência na área e/ou publicar e pesquisar sobre o tema ${ }^{(7)}$.

A construção de um instrumento de triagem para identificar as manifestações fonoaudiológicas na hanseníase pode auxiliar as ações de promoção, prevenção e/ou de reabilitação dos casos e reorientar as ações nas unidades básicas de saúde como forma de melhorar a assistência aos pacientes nos aspectos relacionados às sequelas, contribuindo significativamente para a melhoria da qualidade de vida desses indivíduos. No intuito de atender a essa finalidade, o estudo buscou elaborar um instrumento, contemplando itens relacionados à comunicação e alimentação, incluindo a dimensão da audição, voz, deglutição e fala/expressão facial. A seguir, será descrita a composição final das dimensões do instrumento e a avaliação dos especialistas.

A primeira dimensão, composta pela audição, envolveu apenas um item. Esta recebeu notas máximas por todos os especialistas, obtendo índice de concordância de $100 \%$ para o critério de relevância. $\mathrm{O}$ item classificado como relevante foi o zumbido. Estudo com 30 pacientes diagnosticados com hanseníase - tomando poliquimioterapia por um período médio de seis meses - identificou que 23 pacientes $(76,66 \%$; 45 orelhas) com hanseníase tinham deficiência auditiva neurossensorial; destes, dez $(43,47 \% ; 19$ orelhas) tinham comprometimento neurossensorial leve; dez pacientes $(43,47 \%$; 20 orelhas) apresentaram deficiência auditiva neurossensorial moderada; dois pacientes $(8,69 \%$; quatro orelhas) apresentaram moderada à grave e um paciente apresentou (4,34\%; duas orelhas) deficiência auditiva neurossensorial severa ${ }^{(8)}$. Os estudos na área afirmam que a perda de audição predominante em pacientes que sofrem de doença de Hansen é de origem coclear ${ }^{(8,9)}$.

Os problemas auditivos, encontrados no indivíduo com hanseníase, são consequência do comprometimento do nervo vestibulococlear, levando a sintomas de vertigem, tontura e/ou náusea, à perda auditiva neurossensorial com presença de zumbido e dificuldade de compreensão de fala $^{(10)}$. Já um estudo de 1999 não demonstrou alterações do reflexo estapediano devido à lepra ${ }^{(11)}$.

A segunda seção, composta pela voz, sinalizou dois itens: rouquidão e esforço para falar. Esta dimensão também obteve notas de concordância de $100 \%$ por parte dos especialistas. A doença de Hansen pode ser encontrada em toda a superfície do corpo, incluindo a laringe ${ }^{(12)}$. Estudo realizado na Espanha identificou uma paciente de 28 anos com hanseníase que apresentava disfonia e dispnéia progressiva ${ }^{(12)}$. Pesquisa realizada no Brasil com 13 pacientes com hanseníase detectou que 30,76\% fizeram referência à rouquidão e $46,15 \%$ notaram alguma alteração de sua voz após o uso de medicamentos para a hanseníase ${ }^{(13)}$. Houve presença de pigarro e rouquidão pós-tratamento da hanseníase em idosos ${ }^{(14)}$.

O envolvimento do nervo vago provoca alterações da sensibilidade e motricidade faríngea e laríngea (aspiração e imobilidade de pregas vocais, por exemplo), causando problemas vocais, paralisia do véu palatino e alteração da deglutição ${ }^{(15)}$. As alterações vocais e da deglutição também podem advir das lesões na cavidade oral, cujos sítios mais comumente envolvidos são lábios, palato mole, palato duro, úvula, gengivas, língua e arco palatoglosso, e das lesões na orofaringe que podem estender-se às tonsilas palatinas, parede posterior da faringe e nasofaringe ${ }^{(16,17)}$. As lesões hansênicas, quando ocorrem na cavidade bucal, evoluem de forma insidiosa e assintomática, formando nódulos eritematosos ou amarelados, geralmente múltiplos, principalmente no palato duro. Os dois terços anteriores da língua podem demonstrar atrofia por regressão papilar e áreas nodulares infiltradas. Complicações locais como úlceras e perfurações nasopalatinas eventualmente se estabelecem em decorrência das reações hansênicas ${ }^{(18)}$.

Além disso, quando o Mycobacterium leprae atinge o nervo acessório, ocorre um prejuízo motor e sensitivo da ação da musculatura cervical, resultando em alterações vocais e, caso o nervo hipoglosso seja afetado, ocorre diminuição ou paralisia da língua ${ }^{(19)}$.

Apesar da escassez da literatura sobre essa temática, observamos que esta traz alguns dados sobre alteração da deglutição. Assim, a terceira seção composta pela deglutição abordou cinco itens: dificuldade para mastigar, engasgos, tosse durante a alimentação, dificuldade para engolir e sensibilidade quando o alimento está quente ou frio. Esta dimensão também obteve notas de concordância de $100 \%$ por parte dos especialistas.

Uma pesquisa com 43 pacientes com manifestações clínicas de hanseníase virchowiana identificou que 12 participantes se apresentaram disfágicos, sendo $21 \%$ com disfagia leve, $4,6 \%$ com disfagia moderada e $2,4 \%$ disfagia grave $^{(20)}$. Outro estudo sobre achados fonoaudiológicos na hanseníase observou que, dos 13 pacientes, 69,23\% faziam uso de água (alternância de consistência durante a alimentação), 38,46\% engasgavam com facilidade; $15,38 \%$ apresentavam tosse/pigarro após a deglutição ${ }^{(13)}$.

Outros dados relataram a presença de nódulos granulomatosos no palato mostrando a manifestação bucal em um paciente de hanseníase ${ }^{(16)}$ e presença de lesões nodulares assintomáticas na cavidade bucal de um homem de 55 anos de idade com hanseníase virchowiana ${ }^{(21)}$. A maior parte dos estudos sobre as lesões mucosas na hanseníase se refere àquelas observadas nos pacientes virchovianos. Nestes casos, tem sido descritas lesões no palato duro, mole, arcos palatoglosso e palatofaríngeo, úvula, dorso da língua, mas não são detectadas porque a cavidade bucal raramente 
é examinada ${ }^{(22,23)}$. Os dados confirmam a necessidade do diagnóstico precoce dessas lesões, pois podem levar a prejuízos das funções orais ${ }^{(16)}$.

As funções orais de mastigação e deglutição também podem ser prejudicadas devido ao acometimento do nervo trigêmeo pelo bacilo que provoca diminuição da força da musculatura da mastigação, perda da sensibilidade facial, perda da sensibilidade geral dos $2 / 3$ anteriores da língua, tendo como consequências fonoaudiológicas: disfunção da articulação temporomandibular, assimetria facial, dificuldade mastigatória ${ }^{(24)}$.

A quarta seção, composta pela dimensão da fala/ expressão facial, abordou dois itens: dificuldade na fala e dificuldade em fazer caretas. Esta dimensão também obteve notas de concordância de $100 \%$ por parte dos especialistas.

Quando a hanseníase acomete o nervo facial, provoca perda ou diminuição na movimentação da mímica facial, perda da gustação $2 / 3$ anteriores da língua, alteração da função do músculo bucinador, levando à dificuldade de expressão facial ${ }^{(25-27)}$ e de fala ${ }^{(28,29)}$. O comprometimento dos ossos maxilares e nervos faciais, juntamente com a infecção das mucosas da nasofaringe e orofaringe, resultam em importantes sequelas aos pacientes com hanseníase, tanto cosméticas quanto sociais ${ }^{(18)}$.

Pesquisa enfatizou a relação entre hanseníase e problemas na fala ao descrever que, de 13 indivíduos com hanseníase avaliados, 53,84\% apresentaram dificuldade para falar e $46,15 \%$ notaram diferença após o início do tratamento com polioquimioterapia ${ }^{(13)}$.

\section{CONCLUSÃO}

O protocolo de triagem foi considerado um instrumento válido para identificar as manifestações fonoaudiológicas na hanseníase.

\section{AGRADECIMENTOS}

À Fundação Cearense de Apoio ao Desenvolvimento Científico e Tecnológico (FUNCAP), por meio do Programa Pesquisa para o SUS: gestão compartilhada em saúde, conforme edital 07/2013. Aos juízes, pela disponibilidade e contribuições com o estudo.

\section{REFERÊNCIAS}

1. Zanin LE, Albuquerque IMN, Melo DH. Fonoaudiologia e estratégia de saúde da família: o estado da arte. Rev CEFAC. 2015;17(5):1674-88.

2. Machado RR, Costa E, Erdmann AL, Albuquerque GL, Ortiga AMB. Entendendo o pacto pela saúde na gestão do SUS e refletindo sua implementação. Rev Eletrônica Enferm [Internet]. 2009 [acesso em 2016 Mar 20];11(1):181-7. Disponível em: https://www.fen. ufg.br/fen_revista/v11/n1/pdf/v11n1a23.pdf

3. Jacobi JS, Levy DS, Silva LM. Disfagia: avaliação e tratamento. Rio de Janeiro: Revinter; 2003.

4. Ministério da Saúde (BR), Secretaria de Atenção à Saúde, Departamento de Atenção Básica. Vigilância em saúde: dengue, esquistossomose, hanseníase, malária, tracoma e tuberculose. $2^{\mathrm{a}}$ ed. rev. Brasília: Ministério da Saúde; 2008. (Caderno de Atenção Básica, nº 21).

5. Lanza FM , Vieira NF, Oliveira MMC, Lana FCF. Instrumento para avaliação das ações de controle da hanseníase na Atenção Primária. Rev Bras Enferm. 2014;67(3):339-46.

6. Polit DF, Beck CT. The content validity index: are you sure you know what's being reported? Critique and recomendations. Res Nurs Health. 2006;29(5):489-97.

7. Coluci MZO, Alexandre NMC, Milan D. Construção de instrumentos de medida na área da saúde. Ciênc Saúde Coletiva. 2015;20(3):925-36.

8. Rawlani S, Patil CY, Bhowte R, Degwekar S, Rawlani $\mathrm{S}$, Chandak R, et al. Evaluation of hearing impairment in leprosy patients taking multidrug therapy. Indian J Lepr. 2013;85(4):171-6.

9. Koyuncu M, Celik O, Inan E, Ozturk A. Doppler sonography of vertebral arteries and audiovestibular system investigation in leprosy. Int $\mathrm{J}$ Lepr Other Mycobact Dis. 1995;63(1):23-7.

10. Rodrigues RC. Hanseníase: características auditivas e epidemiológicas [dissertação]. Recife: Fundação Oswaldo Cruz, Centro de Pesquisas Aggeu Magalhães, Departamento de Saúde Coletiva; 2005.

11. Lemieux L, Cherian TA, Richard B. The stapedial reflex as a topographical marker of proximal involvement of the facial nerve in leprosy. A pilot study. Lepr Rev.1999;70(3):324-32.

12. Obando RA, García León N, Márquez MV. Lepra laríngea. Acta Otorrinolaringol Cir Cabeza Cuello. 2012;40(1):71-3.

13. Pereira EC, Almeida LS, Luccia G. Alterações fonoaudiológicas em pacientes portadores de Hanseníase. Rev Univago. 2013;9(1):8-12.

14. Palheta FX Neto, Silva M Filho, Pantoja JMS Junior, Teixeira LLC, Miranda RV, Palheta ACP. Principais queixas vocais de pacientes idosos pós-tratamento para hanseníase. Braz J Otorhinolaryngol. 2010;76(2): $156-63$. 
15. Reichart PA, Srisuwan S, Metah D. Lesions of the facial and trigeminal nerve in leprosy. An evaluation of 43 cases. Int J Oral Surg. 1982;11(1):14-20.

16. Ghosh S, Gadda RB, Vengal M, Pai KM, Balachandran C, Rao R, et al. Oro-facial aspects of leprosy: report of two cases with literature review. Med Oral Patol Oral Cir Bucal. 2010;15(3):459-62.

17. Sánchez Legaza E, Guerrero Cauqui R. Lepra orofaríngea. Acta Otorrinolaringol Cir Cabeza Cuello. 2013;40(4):325-7.

18. Costa MRSN. Considerações sobre o envolvimento da cavidade bucal na hanseníase. Hansen Int 2008;33(1):41-4.

19. Martins ACC, Miranda A, Oliveira MLW, BührerSékula S, Martinez A. Nasal mucosa study of leprosy contacts with positive serology for the phenolic glycolipid 1 antigen. Braz J Otorhinolaryngol. 2010;76(5):579-87.

20. Villar VM, Furia CL, Mello EJ Junior. Disfagia orofaríngea em indivíduos portadores de hanseníase. Rev CEFAC. 2004;6(2):151-7.

21. Dhawan AK, Verma P, Sharma S. Oral lesions in leprosy revisited: a case report. Am J Dermatopathol. 2012;34(6):666-7.

22. Opromolla DVA, Opromolla MA, Ura S. Lesões dimorfas na cavidade oral. Hansen Int. 2003;28(2):1515 .

23. Thomas M, Emmanuel M. A case of advanced lepromatous leprosy with rhino-oro-laryngological involvement in the post-elimination era. Indian J Lepr. 2009;81(2):81-2.

24. Quintas VG, Salles PV, Costa VC, Alvarenga EA, Miranda ICC, Attoni TM. Achados fonoaudiológicos na hanseníase: considerações teóricas. Rev Soc Bras Fonoaudiol. 2009;14(3):560-4.
25. Lalla R, Mulherkar RV, Misar PV. Incomplete peripheral facial nerve palsy and ulnar neuropathy due to leprosy mistaken as faciobrachial stroke. BMJ Case Rep. 2015; 2015.

26. Malhotra HS, Garg RK, Goel MM, Jain A, Gupta A, Lalla $\mathrm{R}$, et al. Bilateral facial synkinesis in leprosy. BMJ Case Rep. 2012;2012.

27. Richard BM, Jacobs JM. Facial nerve pathology in leprosy: searching for the proximal extent of the lesion in facial nerve biopsies. Lepr Rev. 1999;70(3):333-44.

28. Ramos JMH, Souto FJD. Incapacidade pós-tratamento em pacientes hansenianos em Várzea Grande, Estado de Mato Grosso. Rev Soc Bras Med Trop. 2010;43(3): 293-7.

29. Britton WJ. The management of leprosy reversal reactions. Lepr Rev 1998;69(3):225-34.

\author{
Endereço do primeiro autor: \\ Loise Elena Zanin \\ Avenida John Sanford, 1505 \\ Bairro: Junco \\ CEP: 62030-340 - Sobral - CE - Brasil \\ E-mail: loisefono@gmail.com
}

\section{Endereço para correspondência:}

Daniel Hardy Melo

Avenida Comandante Maurocélio Rocha Ponte, 100

Bairro: Derby

CEP: 62042-280 - Sobral - CE - Brasil

E-mail: danielhardy@uol.com.br 